\title{
Speckle Reduction Using Multiple Tones of Illumination
}

\author{
Nicholas George and Atul Jain
}

\begin{abstract}
The occurrence and smoothing of speckle are studied as a function of the line width for a highly collimated illuminating source. A general theory is presented for speckling in the image of a partially diffuse, phase type of object, which has a variable number of random scattering centers per resolution element. Then, an expression is derived for the wavelength spacing required to decouple the speckle patterns arising from two monochromatic tones in an imaging system, thereby establishing that it is feasible to smooth speckle using multicolor illumination. This theory is verified in a series of experiments using both laser illumination and band-limited light from a carbon arc. With highly collimated sources, we show that speckle appears laserlike for an imaged diffuser even up to line widths of $5 \AA$. Then, smoothing of speckle is demonstrated in the imaging of a diffuser and for a section of an optic nerve when the illumination is provided by six narrow lines spread over $1500 \AA$. Since with color-blind, panchromatic viewing the speckle smooths, a direct extension of this method to holographic microscopy, using a multitone laser, should permit one to record and reconstruct holograms of diffraction-limited resolution that are essentially speckle-free.
\end{abstract}

\section{Introduction}

Under monochromatic illumination, objects with a scale of roughness grossly on the order of the wavelength are hard to discern in feature detail, owing to the rapid spatial variations that occur in the scattered radiation. This characteristic of laser illumination to speckle has been studied by many investigators; however, owing to space limitations, we cite only a few of the publications. ${ }^{1-22}$ It is difficult to quote a specific numerical value for this resolution loss since it varies widely with the roughness of the object being studied. However, in the application of laser holography to microscopy, this speckle effect has been a severe obstacle, ${ }^{8-10,12,17,20}$ limiting the working resolution to from a few to several times the classical optics limit. As examples, Young et al. report that "the usual resolution criterion should be divided by five or more whenever diffused laser light is used"12; Close reports resolutions of a few microns on test samples ${ }^{17}$; and Cox et al. have obtained similar resolutions with biological specimens. ${ }^{10}$

Gabor ${ }^{14}$ has classified speckle into two categories: The objective speckle that arises owing to uneven illumination falling on the subject. The subjective speckle that arises from the roughness of the subject in conjunction with the convolving effect of a finite aperture. The objective type can occur when one holographically records a smooth transparency but

The authors are with the California Institute of Technology, Pasadena, California 91109.

Received 25 September 1972. with a diffuser placed in the beam illuminating the transparency. While the diffuser creates a helpful redundancy in the recording, it also leads to the deleterious speckle. Most prior studies of speckle elimination have considered only this objective type, and good results have been reported, although the subject is far from closed. . $^{5,14,22}$

In this paper we consider the smoothing of subjective speckle. This is a somewhat neglected topic, since it has been generally argued that the only effective means for smoothing this type is to increase the aperture. ${ }^{14}$ However, if one draws this conclusion, it is implicitly assumed that operation is at a single wavelength or that separate, independent looks are not being made in the over-all process. In the method we are to describe, separate wavelengths are used to provide independent looks. ${ }^{20,21}$ In this way, we will show that one can smooth subjective speckle at a fixed value of aperture. Probably, too, a method that smooths subjective speckle also smooths the objective type (but not conversely). Hence, in this instance, the need for making the distinction is not great.

An analysis for the wavelength variation of speckle in an imaging system has not been found in the literature; however, Goodman has treated the related problem of the wavelength sensitivity of speckle in the far-field region of a coherently illuminated group of scatterers. ${ }^{2}$ Recent experiments have been reported in confirmation of the thesis that speckle patterns vary spatially in a marked way with change in wavelength. ${ }^{19-21}$

We feel that an interesting possibility for dramati- 
cally reducing the speckle effect in holographic microscopy is to use a multicolor recording process with several widely spaced laser tones spanning the visible optical region. The hologram-volume effects permit one to record and play back these images in a noninteracting way, provided that adequate spatial separation and wavelength intervals have been chosen between each reference beam. ${ }^{23}$ In viewing, the same multitone spectra are used for the reference beams so that no spatial distortions occur, but a color-blind monitor with uniform response over the visible spectrum must be provided, e.g., by a vidicon apparatus, in order to average the multitone speckle.

Within this context, we report the first study of the spatial variations of speckle in the imaging system of a microscope as the wavelength of a monochromatic source is scanned through the visible. First, we analyze the imaging of a diffuser in which there is a variable number of random phase heights per resolution cell. This is a good model for the objects of practical interest covering the smooth case, through the troublesome case where five to ten random scatters per resolution element are limiting the resolution, and the case where the number of random contributors per resolution cell is very high. We show that the multicolor speckle will decorrelate when

$$
\begin{aligned}
\lambda_{2}-\lambda_{1} \geqq\left(\lambda_{0}{ }^{2} / 2 \pi n_{3} h_{0}\right)\left\{\left[1-e^{-\left(p h_{0}\right)^{2}}\right] /[1\right. \\
\left.\left.+(N-1)\left(p h_{0}\right)^{2} e^{-\left(p h_{0}\right)^{2}}\right]\right\}^{1 / 2},
\end{aligned}
$$

where the $n_{3}$ is the difference in the refractive index of the air and the diffuse object, $N$ is the number of scatterers per resolution cell, and the heights $h_{r}$ of the scatterers have been assumed to have a normal distribution with an expected value of zero and a standard deviation $h_{0}, p=-2 \pi n_{3} / \lambda_{0}$, and $\lambda_{0}=\left(\lambda_{1}\right.$ $\left.+\lambda_{2}\right) / 2$. As an alternative interpretation, speckle will appear laserlike as a given line width increases from the few hertz band up to a small fraction of the above $\lambda_{2}-\lambda_{1}$ (this fraction is typically a few angstroms). We note too that wavelength diversity may also prove useful in electron microscopy where the monoenergetic electrons typically have a monochromaticity $\mathrm{d} \lambda / \lambda \lesssim 10^{-5}$, where $\lambda$ is the de Broglie wavelength of the electron. This is easily small enough to cause speckle, however a further consideration of speckle in matter waves is beyond the scope of this paper.

\section{Analysis of Diffuser Imaging}

An imaging system for a microscope is idealized by a lens of focal length $F$ and aperture $D$, an input plane $(\xi, \eta)$ at a distance $s^{\prime}=F+\delta$ from the lens (Fig. 1), and thus with the image plane at a distance $s$ given by $1 / s+1 / s^{\prime}=1 / F$. For monochromatic illumination, $e^{i \omega t}$, of an arbitrary object $D_{0}$, we describe the transverse scalar component of the input electric field by $f(\xi, \eta) e^{i \omega t}$. In the image plane, this corresponding field amplitude is found by two applications of the usual Fresnel-zone approximation of Sommerfeld's formula ${ }^{24}$; i.e., the output field amplitude $E(x, y) e^{i \omega t}$ is given by

$$
\begin{aligned}
E(x, y)= & -\frac{\exp \left[-\left(i 2 \pi / \lambda_{0}\right)\left(s+s^{\prime}\right)\right]}{\lambda_{0}^{2} s s^{\prime}} \\
& \times \iiint \int_{-\infty}^{\infty} d \xi d \eta d u d v f(\xi, \eta) T(u, v) \\
& \times \exp \left\{-\frac{i \pi}{\lambda_{0} s^{\prime}}\left[(u-\xi)^{2}+(v-\eta)^{2}\right]\right. \\
& \left.\quad-\frac{i \pi}{\lambda_{0} s}\left[(x-u)^{2}+(y-v)^{2}\right]\right\},
\end{aligned}
$$

in which $(u, v)$ are Cartesian coordinates in the plane of the lens, $\lambda_{0}=2 \pi c / \omega$, and $c=3 \times 10^{8} \mathrm{~m} / \mathrm{sec}$. The transmission function for the lens $T(u, v)$ will be taken with the spherical convergence factor exp $\left[+\left(i \pi / \lambda_{0} F\right)\left(u^{2}+v^{2}\right)\right]$ and a pupil function that is Gaussian, i.e., it is given by

$$
T(u, v)=\exp \left[-\left(i \pi / \lambda_{0}\right)\left(u^{2}+v^{2}\right) / \rho\right],
$$

where

$$
1 / \rho=-(1 / F)-\left(i 4 \lambda_{0} / \pi D^{2}\right),
$$

and $D$ is the diameter of the lens of focal length $F .^{25}$ Substitution of Eq. (2) into Eq. (1) and integration over the $(u, v)$ plane, defining the magnification factor $M=s / s^{\prime}$ and the up-scaled variables $x^{\prime}=-M \xi$, $y^{\prime}=-M \eta$, give the result:

$$
\begin{aligned}
& E(x, y)= \\
& -\frac{\exp \left\{-\left(i 2 \pi / \lambda_{0}\right)(M+1) s^{\prime}-\left(i \pi / \lambda_{0}\right)\left[\left(x^{2}+y^{2}\right) / M s^{\prime}\right]\right\}}{\lambda_{0}^{2} s^{\prime 2} M^{3}} \\
& \quad \times \frac{\pi D^{2}}{4} \iint_{-\infty}^{\infty} d x^{\prime} d y^{\prime} f\left(-\frac{x^{\prime}}{M},-\frac{y^{\prime}}{M}\right) \\
& \exp \left\{-\frac{i \pi}{\lambda_{0} s^{\prime}}\left[\frac{\left(x^{\prime 2}+y^{\prime 2}\right)}{M^{2}}\right]-\left(\frac{\pi D}{2 \lambda_{0} s}\right)^{2}\left[\left(x-x^{\prime}\right)^{2}\right.\right. \\
& \left.\left.+\left(y-y^{\prime}\right)^{2}\right]\right\} .
\end{aligned}
$$

In the basic imaging equation, Eq. (3), the convolving effect of a finite aperture, $D$, is readily seen. As is well known, subjective speckle arises from this averaging or smearing of the input function $f\left(-x^{\prime}\right)$ $\left.M,-y^{\prime} / M\right)$, i.e., for a finite aperture $E(x, y)$ will not be a perfectly resolved scaled replica of $f\left(-x^{\prime} / M\right.$, $-y^{\prime}(M)$. The departures here from the usual imag-

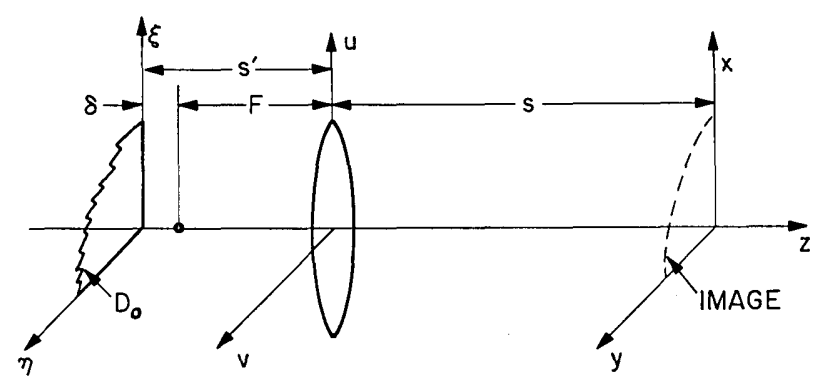

Fig. 1. Single lens magnification with object plane $(\xi, \eta)$ and image plane $(x, y)$. 

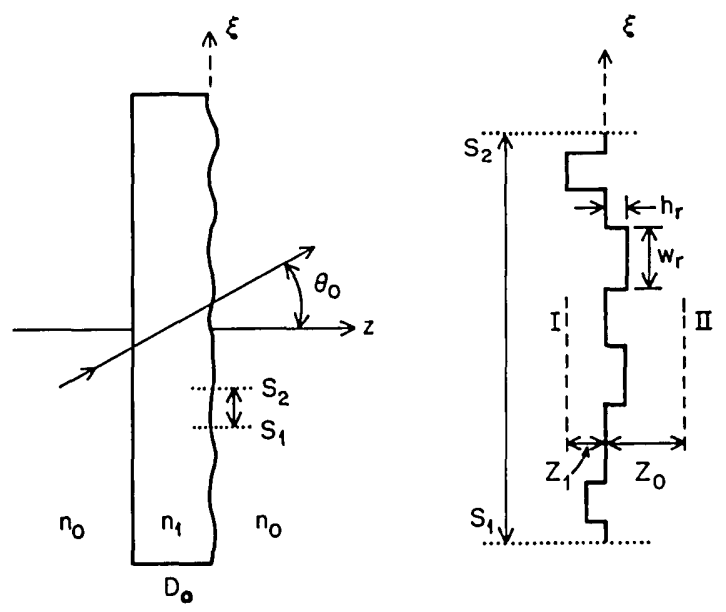

Fig. 2. The idealized diffuser object $\left(D_{0}\right)$ in the $(\xi, \eta)$ plane showing a magnified inset between $\mathrm{S}_{1} \mathrm{~S}_{2}$ for the computation of the transmission function, Eq. (10). The diffuser has steps of width $w_{r}$ and random height $h_{r}$.

ing formula are that phase terms have been retained and the Gaussian transmission function of Eq. (2) is used. Hence, it is instructive to find the radius of the resolution cell for comparison to the conventional circular pupil function. By integration of Eq. (3) with a delta function impulse as input, i.e., $f=$ $\delta\left(x^{\prime}, y^{\prime}\right)$, we find that the output spot size is given by

$$
\dot{E}(x, y) \propto \exp \left[-\left(\pi D / 2 \lambda_{0} s\right)^{2}\left(x^{2}+y^{2}\right)\right] .
$$

Thus, in the output plane $(x, y)$, the intensity falls to a $1 / e^{2}$ fraction of its peak at a radius of $0.637 \lambda_{0} s / D$. From this impulse interpretation, we define the radius of the resolution cell referenced to the $(x, y)$ plane or the $(\xi, \eta)$ plane, respectively, as

$$
\Delta w=\left(2 \lambda_{0} s / \pi D\right) \text { or } \Delta w_{0}=\left(2 \lambda_{0} s^{\prime} / \pi D\right) .
$$

If we would have used the usual circ $\left[\left(u^{2}+\right.\right.$ $\left.v^{2}\right)^{1 / 2} /(D / 2)$ ] as the pupil function portion of Eq. (2a), the resulting Airy disk would have the function value $\left[2 J_{1}(z) / z\right]^{2}$. This would result in a radius for the $1 / e^{2}$ power down locus of $0.82 \lambda_{0} s / D$, which is close enough for our purposes to the corresponding value of $0.637 \lambda_{0} s / D$, obtained for a Gaussian pupil function. ${ }^{26}$

\section{Model for the Phase-Type Diffuser}

There have been various ways, in the past, of describing the electric field transmitted by a rough object. Hopkins and Tiziani, ${ }^{16}$ for instance, idealize the diffuser as a series of closely packed lenslets of varying sizes and focal length. Enloe ${ }^{4}$ and Goodman, ${ }^{2}$ on the other hand, idealize the diffuser as a randomly spaced array of inifinitesimal radiators, each radiating with a random phase.
A semitransparent object of complex-valued amplitude transmission factor $D_{0}(\xi, \eta)$ is placed in the input plane and illuminated by a monochromatic plane wave incident at the polar angle $\theta_{0}$ in the $(\xi, \eta)$ plane (Fig. 2). Thus, the input amplitude function $f(\xi, \eta)$ is given by

$$
f(\xi, \eta)=D_{0}(\xi, \eta) \exp \left[-i\left(2 \pi / \lambda_{0}\right) n_{0} \xi \sin \theta_{0}\right]
$$

where $n_{0}$ and $n_{1}$ are the relative indices of refraction in the two media and $n_{0} \sin \theta_{0}=n_{1} \sin \theta_{1}$. For a general object consisting of an amplitude transmittance, denoted by real-valued function $D_{1}(\xi, \eta)$ and the phase delay $\psi(\xi, \eta)$, we write

$$
D_{0}(\xi, \eta)=D_{1}(\xi, \eta) \exp [-i \psi(\xi, \eta)]
$$

For the surface contour $h(\xi, \eta)$ of the diffuser (shown between planes I and II spaced by $Z_{0}+Z_{1}$ in Fig. 2), we use a ray optics approximation to $\psi(\xi, \eta)$. Thus, we write the phase delay

$$
\begin{aligned}
\psi(\xi, \eta)=\left(2 \pi n_{0} / \lambda_{0}\right)\left(Z_{0}-h\right) / & \cos \theta_{0} \\
& +\left(2 \pi n_{1} / \lambda_{0}\right)\left(Z_{1}+h\right) / \cos \theta_{1} ;
\end{aligned}
$$

and suppressing the nonessential constant phase delays, we can rewrite Eq. (5) as follows:

$$
\begin{aligned}
& f(\xi, \eta)=D_{1}(\xi, \eta) \\
& \exp \left[-\left(i 2 \pi / \lambda_{0}\right) n_{0} \xi \sin \theta_{0}\right] \exp \left[-\frac{i 2 \pi}{\lambda_{0}} n_{3} h(\xi, \eta)\right]
\end{aligned}
$$

in which $n_{3}=\left(n_{1} / \cos \theta_{1}-n_{0} / \cos \theta_{0}\right)$.

Now, consider the simplified one-dimensional case with a pure phase object, i.e., $D_{1}=1$, consisting of randomly positioned steps of height $h_{r}$ and width $w_{r}$ centered at $\xi=\xi_{r}$, i.e.,

$$
h(x, y)=\sum_{r} h_{r} \operatorname{rect}\left[\left(\xi-\xi_{r}\right) / w_{r}\right] .
$$

Then, the one-dimensional idealization of Eq. (7) becomes

$$
\begin{aligned}
f(\xi)=\exp \left[-\left(i 2 \pi / \lambda_{0}\right) n_{0} \xi \sin \theta_{0}\right] \exp \left\{-\left(\frac{i 2 \pi}{\lambda_{0}}\right)\right. \\
\left.n_{3} \sum_{r} h_{r} \operatorname{rect}\left[\left(\xi-\xi_{r}\right) / w_{r}\right]\right\},
\end{aligned}
$$

where the function $\operatorname{rect}(x)=1$ when $|x|<1 / 2$ and is zero otherwise. If nonoverlapping steps are assumed in the expression

$$
\sum_{r} h_{r} \operatorname{rect}\left[\left(\xi-\xi_{r}\right) / w_{r}\right]
$$

one can prove the following identity:

$$
\begin{aligned}
& \exp \left\{-\left(i 2 \pi / \lambda_{0}\right) n_{3} \sum_{r} h_{r} \operatorname{rect}\left[\left(\xi-\xi_{r}\right) / w_{r}\right]\right\}=1 \\
& \quad+\sum_{r} \operatorname{rect}\left[\left(\xi-\xi_{r}\right) / w_{r}\right]\left\{\exp \left[-\left(i 2 \pi / \lambda_{0}\right) n_{3} h_{r}\right]-1\right\} .
\end{aligned}
$$

Substituting this into Eq. (8) we obtain 
$f(\xi)=\exp \left[-i\left(2 \pi / \lambda_{0}\right) n_{0} \xi \sin \theta_{0}\right]$

$\left(1+\sum_{r} \operatorname{rect}\left[\left(\xi-\xi_{r}\right) / w_{r}\right]\left\{\exp \left[-i\left(2 \pi / \lambda_{0}\right) n_{3} h_{r}\right]-1\right\}\right)$.

Equation (9) for a pure phase type of diffuser could equally well have been postulated directly, as follows. The multiplicative term $\exp [-i(2 \pi /$ $\left.\lambda_{0}\right) n_{0} \xi \sin \theta_{0}$ ], with the linear phase taper in $(\xi)$, occurs for a plane wave incident at an angle $\theta_{0}$, as shown in Fig. 2. With nonoverlapping steps assumed in the

$$
\sum_{r} \operatorname{rect}\left[\left(\xi-\xi_{r}\right) / w_{r}\right]
$$

the term within the square brackets, [ ], is either 1 or $\exp \left[-i\left(2 \pi / \lambda_{0}\right) n_{3} h_{r}\right]$. This holds for an arbitrary value of $\xi$, hence $|f(\xi)| \equiv 1$ as it must for a pure phase object.

We could adapt this transmitted field to fit a variety of models for the diffuser. Thus we can consider the height of a step given by $h_{r}(\xi)$ to be roughly constant over the width $w_{r}$. On the other hand, if one prefers the randomly positioned lenslets of Hopkins and Tiziani, then $h_{r}$ becomes the quadratic phase transmission for each lens, i.e., $h_{r}(\xi)=\left[\left(\xi-\xi_{r}\right)^{2} / f_{r}\right]$, where $f_{r}$ is the focal length of the lenslet centered at $\xi_{r}$.

If we make the assumption that the width of each random step is much less than the resolution cell size, i.e., $w_{r} \ll \Delta w_{0}$, then insofar as integrations of the form of Eq. (3), we can replace the rect [( $\xi-$ $\left.\left.\xi_{r}\right) / w_{r}\right]$ by the Dirac delta function, i.e., by $w_{r} \delta(\xi-$ $\left.\xi_{r}\right)$. Thus, combining Eqs. (8) and (9), we find a convenient approximation for the one-dimensional input transmittance

$$
\begin{aligned}
& f(\xi)=\exp \left[-\left(\frac{i 2 \pi}{\lambda_{0}}\right) n_{0} \xi \sin \theta_{0}\right] \\
& \left(1+\sum_{r} w_{r} \delta\left(\xi-\xi_{r}\right)\left\{\exp \left[-\left(i 2 \pi / \lambda_{0}\right) n_{3} h_{r}\right]-1\right\}\right) .
\end{aligned}
$$

To facilitate the consideration of our diffuser, we arbitrarily define the one-dimensional lens process, reducing Eq. (3), as follows:

$$
\begin{array}{r}
E_{1}(x)=\exp \left[-\left(i \pi n_{0} / \lambda_{0}\right)\left(x^{2} / M s^{\prime}\right)\right] \int_{-\infty}^{\infty} d x^{\prime} f\left(-\frac{x^{\prime}}{M}\right) \\
\exp \left\{-\frac{i \pi n_{0}\left(x^{\prime}\right)^{2}}{\lambda_{0} M^{2} s^{\prime}}-\left[\frac{\left(x-x^{\prime}\right)}{\Delta w}\right]^{2}\right\} .
\end{array}
$$

Substitution of Eq. (10) into Eq. (11) and integrating give

$$
\begin{array}{r}
E_{1}(x)=\Delta w(\pi)^{1 / 2} \exp \left[-\frac{i \pi n_{0}}{\lambda_{0}}\left(\frac{x^{2}}{M s^{\prime}}-\frac{2 x \sin \theta_{0}}{M}\right)\right. \\
\left.-\frac{4 \pi n_{0}^{2} x^{2} \sin ^{2} \theta_{0}}{\lambda^{2} M^{2}}\right]+\exp \left[-\frac{i \pi n_{0}}{\lambda_{0}}\left(\frac{x^{2}}{M s^{\prime}}\right)\right] \\
\times \sum_{r} \exp \left[-\left(\frac{x-x_{r}{ }^{\prime}}{\Delta w}\right)^{2}+\frac{i \pi n_{0}}{\lambda_{0}}\left(\frac{2 x_{r^{\prime}} \sin \theta_{0}}{M}-\frac{\left(x_{\left.r^{\prime}\right)^{2}}\right.}{M^{2} s^{\prime}}\right)\right] \\
\times M w_{r}\left[\exp \left(-\frac{i 2 \pi}{\lambda_{0}} n_{3} h_{r_{-1}}\right)-1\right] .
\end{array}
$$

Now define the intensity or energy density factor by $I(x)=E_{1} E_{1} *$, and from Eq. (12) we find

$$
\begin{aligned}
& I(x)=\pi(\Delta w)^{2} e^{-2 \alpha}+2(\pi)^{1 / 2} \Delta w e^{-\alpha} \sum_{r} M w_{r} \\
& \exp \left[-\left(\frac{x-x_{r}{ }^{\prime}}{\Delta w}\right)^{2}\right]\left\{\cos \left(\chi-\phi_{1 r}+\phi_{2 r}\right)-\cos \left(\chi-\phi_{1 r}\right)\right\} \\
& +2 \sum_{r} \exp \left[-2\left(x-x_{r}\right)^{2} /(\Delta w)^{2}\right]\left(M w_{r}\right)^{2}\left\{1-\cos \phi_{2 r}\right\} \\
& +\sum_{\substack{m \\
m \neq r}} \exp \left[-\left(x-x_{m}^{\prime}\right)^{2} /(\Delta w)^{2}-\left(x-x_{r}^{\prime}\right)^{2} /(\Delta w)^{2}\right] \\
& \left.\quad \times \exp \left[i\left(\phi_{1 m}-\phi_{1 r}\right)\right]\right] \\
& \quad \times M^{2} w_{m} w_{r}\left(e^{-i \phi_{2 m}}-1\right)\left(e^{\left.+i \phi_{2 r}-1\right)},\right.
\end{aligned}
$$

in which $\alpha=4 \pi\left(n_{0} x \sin \theta_{0}\right)^{2} /\left(\lambda_{0} M\right)^{2}$,

$$
\chi=\left(2 \pi / \lambda_{0}\right)\left(n_{0} x \sin \theta_{0} / M\right),
$$

$\phi_{1 r}=\left(\pi n_{0} / \lambda_{0}\right)\left\{2 x_{r}^{\prime} \sin \theta_{0} / M-\left(x_{r}^{\prime}\right)^{2} /\left(M^{2} s^{\prime}\right)\right\}$, and

$$
\phi_{2 r}=\left(2 \pi / \lambda_{0}\right) n_{3} h_{r} .
$$

Equation (13) is a convenient starting point for the statistical analysis of decorrelation based on combining intensity patterns taken at separate wavelengths. ${ }^{21}$ Equation (12) is the more useful starting point in the simpler amplitude decoupling criterion presented in Eq. (21) of the following section.

\section{Expected Value and Variance for the Electric Field}

To calculate the wavelength spacing required to decouple the speckle pattern described by Eqs. (12) and (13), first we analyze the simpler case when $\theta_{0}$ is constant, e.g., when $\theta_{0}=0$. Later separate comment is included for the important case when a distributed angular spectrum is used for the illumination.

We assume the response of a panchromatic viewing system is given by $I=E_{1} E_{1} *+E_{2} E_{2} *+\ldots$ when there are multiple tones of monochromatic illumination, i.e., beat terms are negligible with long exposure times. Hence, we must compare the intensities in the image plane at two different wavelengths and find when these two intensity patterns are decorrelated. Since in computing $E_{1} E_{1} *$, common phase terms will cancel, we factor Eq. (12) extracting and suppressing the term $\exp \left\{\left(i \pi n_{0} / \lambda_{0}\right)\left[\left(2 x \sin \theta_{0} /\right.\right.\right.$ $\left.\left.M)-\left(x^{2} / M s^{\prime}\right)\right]\right\}$. The remainder of the electric field we denote by $\mathbf{E}_{1}$, and rewriting Eq. (12) gives

$$
\begin{aligned}
\mathrm{E}_{1}(x) & =\Delta w(\pi)^{1 / 2}+\sum_{r} \exp \left[-\left(\frac{x-x_{r}{ }^{2}}{\Delta w}\right)^{2}\right. \\
& \left.-\frac{i \pi n_{0}\left(x_{r}^{\prime}\right)^{2}}{\lambda_{0} M^{2} s^{\prime}}\right] M w_{r}\left\{\exp \left(-\frac{i 2 \pi}{\lambda_{0}} n_{3} h_{r}\right)-1\right\} .
\end{aligned}
$$

Additionally, comparing Eqs. (12) and (14), one will note that we have dropped the quadratic multiplier $\exp \left[-4 \pi n_{0}^{2} x^{2} \sin \theta_{0} /\left(\lambda_{0}^{2} M^{2}\right)\right]$ from the first term in Eq. (14); and the small phase term $\exp \left\{\left(i \pi n_{0}\right\}\right.$ $\left.\left.\lambda_{0}\right)\left[2\left(x_{r}{ }^{\prime}-x\right) \sin \theta_{0} / M\right]\right\}$ from the summation. 
To study the speckle in the image plane, we consider an arbitrary position $x$. In Eq. (14), the rapid falloff of the Gaussian term, $\exp \left\{-\left[\left(x-x_{r}{ }^{\prime}\right) / \Delta w\right]^{2}\right\}$, limits the number of scatterers from among the entire set $x_{r}{ }^{\prime}$ that contribute effectively to $\mathbf{E}_{1}$ at $x$. Thus, if there are $N$ scatterers remaining from this set that are positioned (in image plane coordinates) with values of $x_{r}{ }^{\prime}$ in the range $x-\Delta w<x_{r}{ }^{\prime}<x+$ $\Delta w$, then we can approximate Eq. (14) by

$$
\mathrm{E}_{1}(x)=A-B N+B \sum_{r=1}^{N} \exp \left(+i p h_{r}\right) .
$$

We have defined $A=\Delta w(\pi)_{r}^{1 / 2}$,

$$
\begin{gathered}
B=M w_{c} \exp \left[-\left(i \pi n_{0} x^{2}\right) /\left(\lambda_{0} M^{2} s^{\prime}\right)\right] \text { and } p \text { as } \\
p=-\left(2 \pi n_{3} / \lambda_{0}\right) .
\end{gathered}
$$

To simplify the analysis, we assume that the step widths $w_{r}$ are constant for all scatterers and equal to $w_{c}$. Also, with negligible error, the Gaussian permits us to approximate the phase term exp $-\left[i \pi n_{0}\left(x_{r}\right)^{2} / \lambda_{0} M^{2} s^{\prime}\right]$ in Eq. (14) by the term exp - $\left[i \pi n_{0} x^{2} / \lambda_{0} M^{2} s^{\prime}\right]$ in Eq. (15).

If we assume that the real random variable $h_{r}$ is distributed according to some known density function, $f\left(h_{r}\right)$, and the corresponding characteristic function for this distribution is given by $F(p)=$ $\int_{-\infty}^{\infty} \exp (+\mathrm{iph}) f(h) d h$, then we also obtain the following results for the expectation and variance of $e^{i p h}$ (Ref. 27):

$$
\begin{aligned}
\left\langle e^{i p h}\right\rangle & =F(p), \\
\sigma^{2}\left(e^{i p h}\right) & =1-F(p) F^{*}(p) .
\end{aligned}
$$

If we also assume that the random variables $h_{r}$ represented by different scattering points are independent, then we can calculate the expected value and variance of our amplitude defined by Eq. (15). Thus, we obtain the following expression for the expected value of the electric field at a point in the image plane

$$
\left\langle\mathrm{E}_{1}(x)\right\rangle=A-N B+B N F(p) .
$$

Also, the variance in this electric field, defined by $\sigma^{2}\left[\mathbf{E}_{1}(x)\right]=\left\langle\left(\mathbf{E}_{1}-\left\langle\mathbf{E}_{1}\right\rangle\right)\left(\mathbf{E}_{1}-\left\langle\mathbf{E}_{1}\right\rangle\right)^{*}\right\rangle$, is readily computed by substitution of Eq (15) into this form and simplification using Eqs. (17)-(19). The resulting expression for the variance in the electrical field is

$$
\sigma^{2}\left[\mathrm{E}_{1}(x)\right]=N\left(1-F F^{*}\right) B B^{*},
$$

where $B B^{*}=\left(M w_{c}\right)^{2}$ and the characteristic function $F$ is given by Eq. (17).

\section{Wavelength Spacing for Speckle Decorrelation}

We note that our expected value is some complex number, while the variance is a real number since it is defined by $\sigma^{2}=\left\langle\left|\mathbf{E}_{1}-\left\langle\mathbf{E}_{1}\right\rangle\right|^{2}\right\rangle$. Thus, Eq. (20) gives us the square of the radius of a circle centered around the expected value within which roughly half of our values of $\mathbf{E}_{1}$ lie. The intensity at some fixed point $x$ can thus be considered to have changed significantly when the magnitude of the change in $\mathbf{E}_{1}(x)$ with wavelength is of the order of the standard deviation. Thus, we adopt the criterion that the speckle is decoupled when the wavelength change causes the average of the magnitude squared change in $\mathbf{E}_{1}(x)$ to be equal to the variance for a particular wavelength $\lambda_{0}$, i.e., decorrelation occurs whenever

$$
\left\langle\Delta \mathrm{E}_{1}(x) \Delta \mathrm{E}_{1}(x)^{*}\right\rangle \geq \sigma^{2}\left[\dot{\mathrm{E}}_{1}(x)\right] .
$$

The change in the electric field $\mathbf{E}_{1}(x)$ with wavelength interval $\Delta \lambda$ is given by

$$
\Delta \mathbf{E}_{1}(x)=\left[\partial \mathbf{E}_{1}(x) / \partial \lambda\right] \Delta \lambda .
$$

Thus differentiating Eq. (15) with respect to $\lambda$ and noting that $d p / d \lambda=\left(+2 \pi n_{3} / \lambda^{2}{ }_{0}\right)$, we obtain

$$
\begin{aligned}
\Delta \mathrm{E}_{1}(x)=\left(-\Delta B N+B \sum_{r=1}^{N} i h_{r} e^{i p h_{r}} \frac{2 \pi n_{3}}{\lambda_{0}^{2}}\right. & \\
& \left.+\Delta B \sum_{r=1}^{N} e^{i p h_{r}}\right) \Delta \lambda,
\end{aligned}
$$

where

$$
\Delta B=i \pi n_{0} x^{2} M w_{c} /\left(\lambda_{0}^{2} M^{2} s^{\prime}\right) \exp \left[i \pi n_{0} x^{2} /\left(\lambda_{0} M^{2} s^{\prime}\right)\right] .
$$

We now consider the speckle near the axis, i.e., when $\Delta B$ is negligible (worst case). In this case Eq. (22) reduces to

$$
\Delta \mathrm{E}_{1}(x)=\simeq B \sum_{r=1}^{N} i h_{r} \frac{2 \pi n_{3}}{\lambda_{0}{ }^{2}} e^{i p h_{r}} \Delta \lambda
$$

If we now compute $\left\langle\mathbf{E}_{1}(x) \Delta \mathbf{E}_{1}(x)^{*}\right\rangle$, we obtain the following result, assuming that the expected value of $h_{r}$ is zero and that the standard deviation is $h_{0}$,

$$
\begin{aligned}
& \left\langle\Delta \mathrm{E}_{1}(x) \Delta \mathrm{E}_{1}(x)^{*}\right\rangle=\left(2 \pi n_{3} \Delta \lambda / \lambda_{0}^{2}\right)^{2}\left\{h_{0}^{2} N\right. \\
& \left.\quad+\left(N^{2}-N\right)[(d / d p) F(p)][(d / d p) F(p)]^{*}\right\} B B^{*}
\end{aligned}
$$

Substituting Eqs. (20) and (24) into the criterion, Eq. (21), we obtain a value for the wavelength spacing $\lambda_{2}-\lambda_{1}$ required to decorrelate the speckle:

$$
\begin{aligned}
\lambda_{2} & -\lambda_{1} \\
= & \frac{\lambda_{0}{ }^{2}}{2 \pi n_{3}}\left\{\frac{1-F(p) F^{*}(p)}{h_{0}{ }^{2}+(N-1)[(d / d p) F(p)][(d / d p) F(p)]^{*}}\right\}^{1 / 2}
\end{aligned}
$$

[decoupled case]. (25)

The speckle pattern will therefore be laserlike when the spectral line width of a single tone is much less than is given by Eq. (25), i.e., when the line width $\Delta \lambda$ is given by 


$$
\begin{aligned}
& \Delta \lambda \leq \frac{1}{10}\left(\lambda_{2}-\lambda_{1}\right) . \\
& \leq \frac{\lambda_{0}{ }^{2}}{20 \pi n_{3}}\left\{\frac{1-F(p) F^{*}(p)}{h_{0}{ }^{2}+(N-1)[(d / d p) F(p)][(d / d p) F(p)]^{*}}\right\}^{1 / 2}
\end{aligned}
$$

[laserlike case].

To illustrate the above results with an example, assume that the heights of the scatterers are distributed normally, where our expected value and standard deviation for the heights have already been taken "to be zero and $h_{0}$, respectively. Thus, we write the density function for $h_{r}$ as

$$
f\left(h_{r}\right)=\exp \left[-h_{r}^{2} /\left(2 h_{0}^{2}\right)\right] / h_{0}(2 \pi)^{1 / 2}
$$

and the corresponding characteristic function as

$$
F(p)=\exp \left(-1 / 2 p^{2} h_{0}^{2}\right) .
$$

Substituting Eq. (27) into Eq. (25) we obtain the wavelength spacing required to decorrelate the speckle pattern as

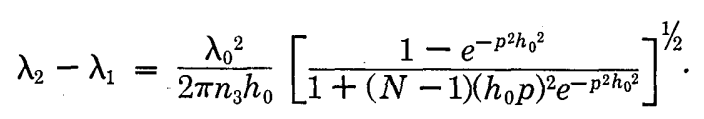

We note that for the case of a very rough diffuser, where $\left(p h_{0}\right)$ is greater than 1, Eq. (28) can be approximated by

$$
\lambda_{2}-\lambda_{1}=\lambda_{0}^{2} /\left(2 \pi n_{3} h_{0}\right), \quad \text { when }\left(p h_{0}\right)^{2} \gg 1 .
$$

In the case of a relatively smooth diffuser, Eq. (28) can be reduced to

$$
\lambda_{2}-\lambda_{1}=\frac{\lambda_{0}}{\left[1+(N-1)\left(p h_{0}\right)^{2}\right]^{1 / 2}}, \text { when }\left(p h_{0}\right)^{2} \ll 1 .
$$

Now assuming monochromatic illumination, we study the condition under which we can expect a particular diffuser to give a large amount of speckle. If we take the ratio of the standard deviation to the amplitude of the expected value, i.e., by Eqs. (19) and (20), we obtain the average fractional change in amplitude among different resolution cells of width $2 \Delta w$. When this ratio is very small, we have the case when most cells have the same intensity; and there is practically no speckle. In the case where this ratio approaches 1 , we have a badly speckled case. Thus, for the normal distribution, as defined by Eq. (27), this ratio is given by

$$
R=\frac{(N)^{1 / 2}\left(1-e^{-p^{2} h_{0}^{2}}\right)^{1 / 2}|B|}{\left|A-N B+N B e^{-p^{2} h_{0}^{2} / 2}\right|} .
$$

By Eq. (31), we note that $R$ goes to zero, i.e., no speckle, when the roughness as characterized by $p h_{0}$ decreases. Alternatively, taking $A$ to be of the same order of magnitude as $N B$, we note that the ratio $R$ is proportional to $1 /(\mathrm{N})^{1 / 2}$. Thus, the badly speckled case occurs with small numbers of scatterers per resolution element. And as $N$ gets very large, say exceeding 100, the amount of speckle is drastically reduced.

If we assume operation at a single wavelength, it is still possible to obtain averaging of speckle by superimposing image intensities formed when the diffuser is illuminated successively by plane waves at different angles of incidence. We can calculate the angular difference $\Delta \theta_{0}$ between successive plane waves incident at angles $\theta_{01}$ and $\theta_{02}$, which will decouple these respective image intensities. Since we are considering the sequential recording of intensities, the phase term containing $\theta_{0}$, which was suppressed in writing Eq. (14), still does not enter: and Eqs. (15) and (16) form a convenient starting point. From $n_{3}=\left(n_{1} / \cos \theta_{1}-n_{0} / \cos \theta_{0}\right)$ and $n_{1} \sin \theta_{1}=$ $n_{0} \sin \theta_{0}$, we compute the angular variation of $n_{3}$ :

$$
\begin{aligned}
d n_{3} / d \theta_{0}=(1 / 2)\left(n_{0}{ }^{2} / n_{1}\right)\left(\sin 2 \theta_{0} / \cos ^{3} \theta_{1}\right) & \\
& -\left(n_{0} \sin \theta_{0} / \cos ^{2} \theta_{0}\right) .
\end{aligned}
$$

The variation of $p$, at fixed wavelength, is found by differentiation of Eq. (16), i.e.,

$$
d p / d \theta_{0}=\left(-2 \pi / \lambda_{0}\right)\left(d n_{3} / d \theta_{0}\right) .
$$

Following the same mathematical procedure as we did in deriving Eq. (25) except that now $\Delta \mathbf{E}_{\mathbf{1}}(x)=$ $\left[\partial \mathbf{E}_{1}(x) / \partial \theta_{0}\right] \Delta \theta_{0}$, one can readily show that the angular spacing $\Delta \theta_{0}=\theta_{02}-\theta_{01}$ is given by

$$
\begin{aligned}
\Delta & \theta_{0} \\
= & \left.\left(\lambda_{0}\left\{\frac{1-F(p) F^{*}(p)}{h_{0}^{2}+(N-1)[(d / d p) F(p)][(d / d p) F(p)]^{*}}\right\}^{1 / 2}\right)\right|^{\prime} \\
& \left\{\pi n_{0}\left[2 \sin \theta_{0} / \cos ^{2} \theta_{0}-n_{0} \sin 2 \theta_{0} /\left(n_{1} \cos ^{3} \theta_{1}\right)\right]\right\} \cdot(34)
\end{aligned}
$$

For the case when the heights are distributed normally, by substitution of Eq. (27) into (34), we find the angular separation required to decouple the speckle patterns reduces to

$$
\begin{aligned}
& \Delta \theta_{0}=\left(\lambda_{0}\left\{\left(1-e^{-p^{2} h_{0}^{2}}\right) /\left[1+(N-1)\left(h_{0} p\right)^{2} e^{-p^{2} h_{0}^{2}}\right]\right\}^{1 / 2}\right) / \\
& {\left[\pi n_{0} h_{0}\left(\frac{2 \sin \theta_{0}}{\cos ^{2} \theta_{0}}-\frac{n_{0} \sin 2 \theta_{0}}{n_{1} \cos ^{3} \theta_{1}}\right)\right] \cdot(3}
\end{aligned}
$$

We will use Eq. (35) in the following section to establish a numerical value for the degree of collimation required to assure that our speckle averaging is due to wavelength variation and not to multiangular illuminating beams.

\section{Experiments with Laser Sources}

Three different experiments were conducted using a laser. In the first, a single mode argon laser was used to illuminate a ground glass diffuser and the speckle positions were charted as the wavelength was set to the various principal lines ( $4579 \AA, 4727 \AA$, $4765 \AA, 4880 \AA, 4965 \AA, 5017 \AA$, and $5145 \AA$ ). In this experiment, the statistical problem is different in the details from the analysis presented herein 
(e.g., see Goodman's treatment ${ }^{2}$ ) in that the number of scatterers that contribute at each point in the output plane is essentially that of the entire illuminated diffuser; and this is usually a very large number. Since, in this experiment, the diffuser is not being imaged, one should not be thinking in terms of the number of scatterers per resolution cell, i.e., the $N$ of the previous section. Nevertheless, speckle repositioning is readily seen; the bright spots in the image vary quite noticeably for the wavelength shifts of 100 $\AA$ or so.

In this first experiment it is also interesting to vary the size of that portion of the diffuser that is being illuminated. The equation for the speckle size in the nonimaged case indicates a speckle size inversely proportional to the entire object dimension. ${ }^{16}$ Thus, one can greatly enlarge the mean speckle size by placing a small aperture at the diffuser. It is an experimental convenience to work with these larger speckles. An effective nonmechanical means for providing this variable aperture is to pass the collimated output of the laser directly through a microscope objective. Then, of course, the spot size of the exit beam converges to a small diameter in the rear focal plane of the objective, and thereafter it rapidly expands. The diffuser is placed in this exit beam; axial translation of the diffuser in this region near and beyond the rear focal plane provides a handy method for varying the effective aperture over a wide range. The diffuser is fixed at a specific position that gives large, easily monitored speckles on a screen at $1-2-\mathrm{m}$ distance; and then the wavelength is varied. Again, no imaging is involved; and the speckle positions are readily charted by directly exposing a piece of sheet film placed at the screen. While speckle repositioning is definitely observed at the different wavelengths, we were not satisfied that we had the amount of control necessary to corroborate the theory, e.g., the wavelength spacing and total interval available were limited.

A more persuasive experimental observation of speckle averaging with multiple tones of monochromatic illumination was obtained in the following second experiment using an argon laser source. The prism line selector, not the single mode etalon, is removed so that oscillation is obtained simultaneously on several of the transitions listed in the preceding paragraph. The speckle averages pretty well for these wavelength spacings; photographing an object illuminated first by a single tone and then by the multitone clearly shows this effect. In this imaging experiment, the aperture of the camera is used to control the amount of subjective speckle that is introduced by the camera lens.

Unfortunately, the spread of wavelengths and the single collimated beam of the argon laser are not adequate for the recording and reconstruction of spec'kle-free holograms as a simple, single exposure process. Both spatial and spectral separation of the multitone reference beams are probably going to be required. ${ }^{23}$
A third experiment was performed using a single mode helium-neon laser at $6328 \AA$. This laser is continuously tunable over the Doppler-broadened transition (about $1200 \mathrm{MHz}$ ) by a piezoelectric control of the cavity length. Using the 3-M Company's Magic Transparent Tape as a standard diffuser, since it is both readily available and reproducible, we carefully measured bright spot positons in speckle patterns as the wavelength was scanned over 1200 $\mathrm{MHz}$. By this experiment, we concluded that the motion is below the threshold of detection for such small shifts in frequency. This null result is in agreement with the prediction of our theory, e.g., Eq. (26). Care was taken to control the angular drift of the laser beam in all of these experiments [see $\theta_{0}$ in Eq. (12)]. This is particularly important for the argon laser since the wavelength selection consists of angularly positioning a prism in the cavity. Both this and the large temperature changes during warmup cause easily measurable drifts in $\theta_{0}$. For our experiments, angular control is obtained by passing the laser beam through two high-quality microscope objectives separated by twice their focal length $(20 \mathrm{~mm})$ with a $5-\mu \mathrm{m}$ diam pinhole located at their common focus. The output beam from this unity beam expander-spatial filter device is used as the source. Thus, the angular deviation in $\theta_{0}$ is maintained at some small portion of $2 \times 10^{-4} \mathrm{rad}$.

\section{Multicolor Speckle Experiments}

A series of experiments was also performed using both the Scotch Magic Tape diffuser and a biological specimen as objects with band-limited light being used to simulate a continuously tunable laser (see Fig. 3). A carbon arc or high-pressure mercury arc

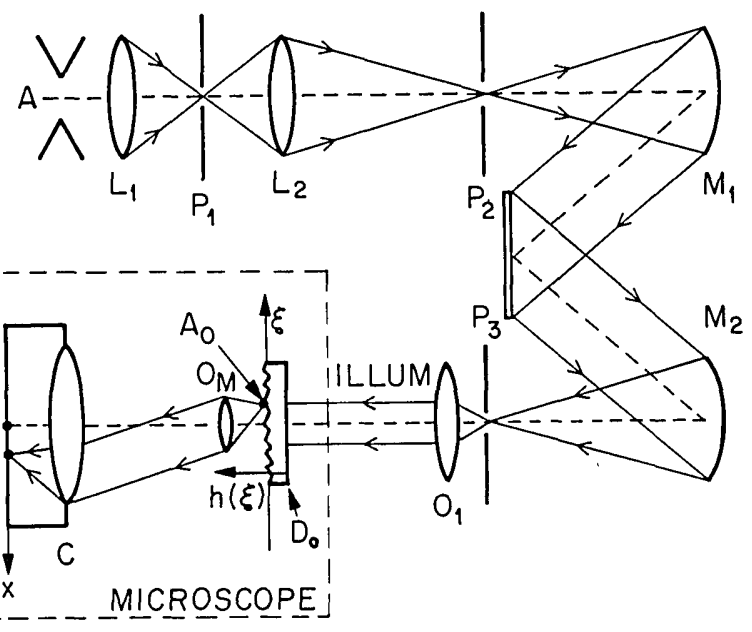

Fig. 3. The experimental arrangement for obtaining laserlike speckle from a band-limited, carbon arc source. The diffuse object $D_{0}$, is magnified by the microscope $O_{M}$ and speckled images are photographed by the camera $C$. Illumination from the arc $(A)$ is band limited by the Spex monochromater (mirrors $\mathbf{M}_{1}$, $\mathrm{M}_{2}$ are $10-\mathrm{cm}$ diam and $75-\mathrm{cm}$ focal length); pinholes $\mathrm{P}_{1}$ and $\mathrm{P}_{2}$ are $400 \mu \mathrm{m}, \mathrm{P}_{3}$ is $60 \mu \mathrm{m}$, and the microscope objective $\mathrm{O}_{1}$ is used to collimate the illumination at $\mathrm{D}_{0}$. 


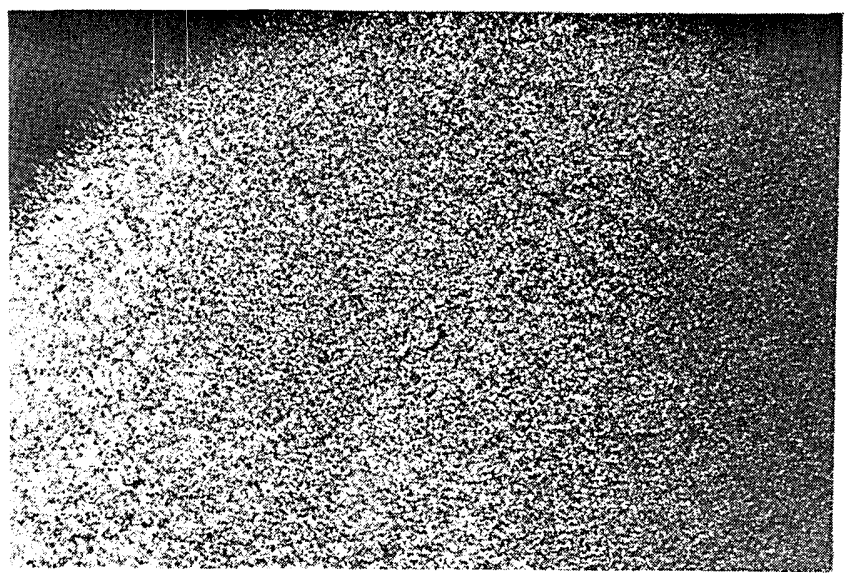

Fig. 4. Speckle pattern with collimated laser illumination at $6328 \AA$ incident on diffuser made from Scotch Magic Tape. Imaging is as shown in Fig. 3.

source (A) is collimated, dispersed by the grating (G) for band limiting by pinholes $\mathrm{P}_{2}, \mathrm{P}_{3}$; then it is highly collimated by the pinhole-objective lens combination $\left(\mathrm{P}_{3}, \mathrm{O}_{1}\right.$ in Fig. 3). The objects are placed at $\mathrm{D}$ for magnification by the microscope, $\mathrm{O}_{\mathrm{M}}$, and photograpbically recorded by the camera (C).

Since the principal objective of this experiment is a detailed study of the averaging of speckle from a multitone source, first we must establish values for the allowable width of the single tone; then, we will take a series of exposures with wavelength differences exceeding the interval given by Eq. (28). The quantitative treatment of the degree of smoothing that results from a number of independent tones is beyond the scope of this paper. ${ }^{21}$

For experimental purposes in the classification of diffusers, it is convenient to recognize that $\left[\Delta w_{0} /\right.$ $\left.\left\langle w_{r}\right\rangle\right]^{2}$ sets an upper limit to the number of samples per resolution cell and that $h_{0}$ and $\left\langle w_{r}\right\rangle$, are coupled, i.e., by coupled, we mean that in making a finer diffuser, $\left\langle w_{r}\right\rangle$ becomes less, but the attainable $h_{0}$ usually decreases too. Hence, we rewrite Eq. (28) as follows:

$$
\begin{aligned}
\frac{\Delta \lambda}{\lambda}= & \frac{\lambda}{2 \pi n_{3} h_{0}}\left(\left\{1-\exp \left[-\left(2 \pi n_{3} h_{0} / \lambda\right)^{2}\right]\right\} /\left\{1+\left[\left(\Delta w_{0} /\right.\right.\right.\right. \\
& \left.\left.\left.\left.\left\langle w_{r}\right\rangle\right)-1\right]\left[h_{0}\left(2 \pi n_{3} / \lambda\right)\right]^{2} \exp \left[-\left(2 \pi n_{3} h_{0} / \lambda\right)^{2}\right]\right\}\right),(36
\end{aligned}
$$

where $\Delta w_{0}=2 \lambda F /(\pi D)$.

The Scotch Magic Tape diffuser is relatively rough; separate measurements using a depth microscope fix the depth $h_{0} \gtrsim 8 \mu \mathrm{m}$, although it could be as high as $14 \mu \mathrm{m}$ and the width $\left\langle w_{r}\right\rangle \approx 1 \mu \mathrm{m}$. For $n_{3}$ $=0.6$ and $\lambda_{0}=0.5 \mu \mathrm{m}$, we find $p h_{0}=50$, hence by Eq. (29) we predict that

$$
\lambda_{2}-\lambda_{1}=80 \AA
$$

decouples, and the speckle should remain laser-like for line widths up to about one tenth of this, i.e., for
$8 \AA$. We note that $N$ does not enter into this computation, since $p h_{0} \gg 1$.

For the spectrometer used, the output line width from $\mathrm{P}_{3}$ in Fig. 3 is just slightly under $5 \AA$ for the input pinhole $\left(\mathrm{P}_{2}\right)$ of $400 \mu \mathrm{m}$ and the exit pinhole $\left(\mathrm{P}_{3}\right)$ of $60 \mu \mathrm{m}$. In the customary language of partial coherence, ${ }^{29}$ an equivalent viewpoint on the need to restrict $\Delta \lambda$, as by Eq. (26), is that we must restrict the temporal coherence so that axial path differences inherent in traversing the diffuser will not smooth out the laserlike speckle. Thus, we have $n_{3} h_{0} \ll$ $c / \Delta \nu$; hence, we see that $n_{3} h_{0} \Delta \lambda / \lambda^{2} \ll 1$, which is precisely the expression used to compute the $8-\AA$ limit in the preceding paragraph.

$\mathrm{P}_{2}$ essentially controls $\Delta \lambda$ in the apparatus; and in a relatively independent manner, the much smaller $\mathrm{P}_{3}$ controls the transverse coherence. This is the reason for the disparity in our choices of their sizes. This, too, can be understood directly from Eq. (13). A larger pinhole $P_{3}$ permits a continuous range of angle $\theta_{0}$ from zero up to some maximum $\theta_{02}$ in the illumination of the sample.

From Eq. (35) and the discussion of the previous section, we obtain the maximum angular bandwidth allowable for laserlike speckle to be

$$
\Delta \theta=\frac{1}{10}\left\{\frac{\lambda \cos ^{2} \theta_{0}}{\pi h_{0}\left[n_{0}-\left(n_{0}^{2} / n_{1}\right)\right]}\right\}^{1 / 2},
$$

in the case of rough diffuser, i.e., $p h_{0} \gg 1$, where $\theta_{0}$ is the mean incident angle. For the Scotch Magic Tape diffuser, this value is $0.02 \mathrm{rad}$. A simple geometrical consideration gives the illumination on the diffuser (D) in Fig. 3 to have an angular bandwidth of $1.9 \times 10^{-3} \mathrm{rad}$, and so we are well within the limit to see speckle. (Parenthetically, we note that when the diffuser is very rough, the occurrence of speckle is reasonably estimated using a simple temporal coherence requirement and the usual transverse coherence requirement, which results from the van Cittert-Zernike theorem. However, in the intermediate

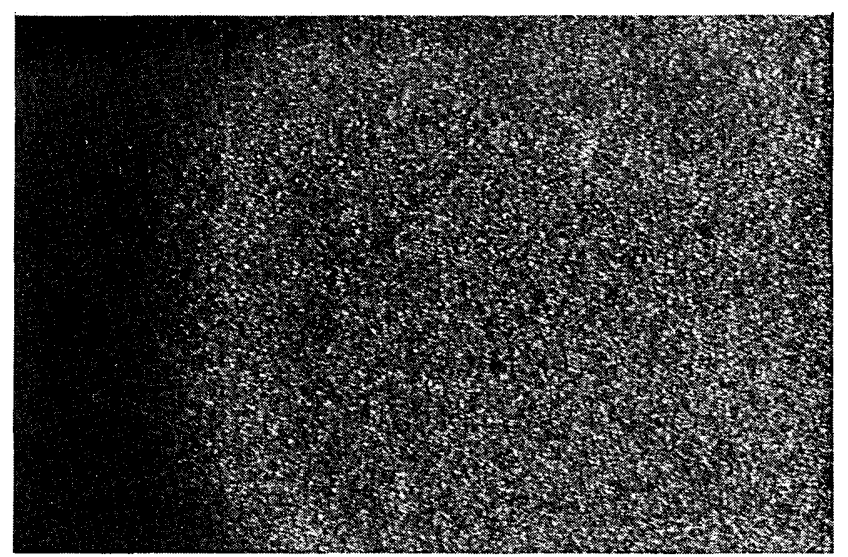

Fig. 5. Speckle pattern for Scotch Magic tape diffuser, as in Fig. 4, but illuminated with band-limited light from a carbon arc (5 $\AA$ band limited at $6000 \AA$ and $70 \mathrm{~min}$ of exposure using Tri-X film). Beam collimation angle is $2 \times 10^{-3} \mathrm{rad}$. 


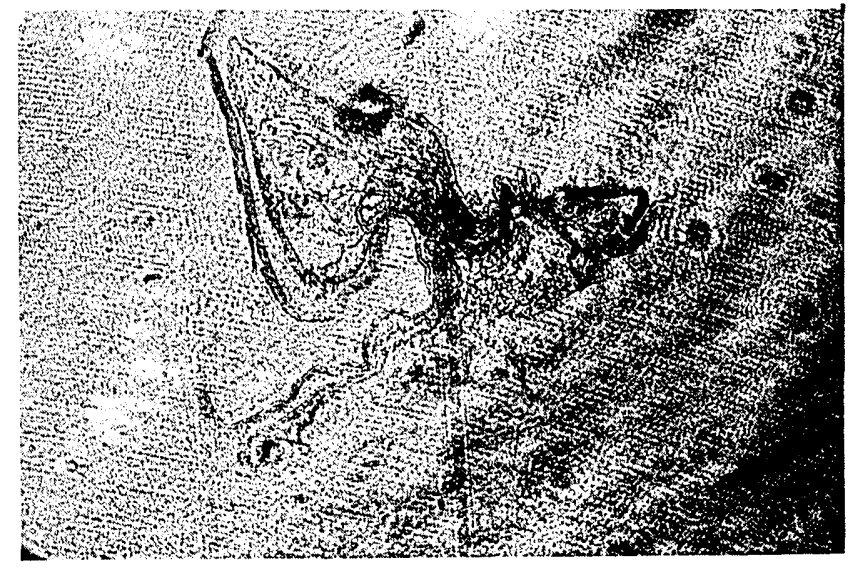

Fig. 6. Section of optic nerve at low magnification illuminated by collimated laser light.

range, $p h_{0} \sim 1$, the statistical properties control, and the formulas of the last section should be used.)

Band limiting the source to a $5 \AA$ width and maintaining adequate transverse coherence greatly reduces the illumination level at the camera. A value for the exposure time is estimated in the following discussion. Referring to Fig. 3, and noting that the energy radiated by the source A (carbon arc lamp) is $10^{-2} \mathrm{~W} \mathrm{sr}^{-1} \AA^{-1}$ and that the $f$ numbers of the lenses $\mathrm{L}_{1}$ and $\mathrm{L}_{2}$ and mirrors $\mathrm{M}_{1}$ and $\mathrm{M}_{2}$ have been matched, we obtain the energy transmitted through the pinhole $\mathrm{P}_{3}$ to be $3 \times 10^{-6} \mathrm{~W}$ in a 5 - $\AA$ bandwidth. The intensity at the diffuser is approximately $2 \times$ $10^{-5} \mathrm{~W} / \mathrm{cm}^{2}$. The magnification of the objective and camera system $\left(\mathrm{O}_{M}\right.$ and $\left.\mathrm{C}\right)$ is 20 and so the intensity of the light hitting the film is $4 \times 10^{-8} \mathrm{~W} /$ $\mathrm{cm}^{2}$. Now the film requires, including the reciprocity loss factor, $50 \mu \mathrm{J} / \mathrm{cm}^{2}$ of energy to be at threshold for recording, and so this gives a minimum exposure time of $20 \mathrm{~min}$.

The speckle pattern using a collimated $6328-\AA$ laser to illuminate the Magic Tape diffuser D in Fig. 3 is shown in Fig. 4. The laserlike speckle pattern using tbe high pressure arc source is shown in Fiq. 5 . Even so, some averaging of the speckle pattern is evident with the highly collimated arc source band limited to $5 \AA$. While a narrower line width would have been desirable, the extremely long exposure times, $70 \mathrm{~min}$ for one tone, forced this compromise. With the arc source illumination, the speckle is also easily seen visually through the microscope eyepiece (after 15 min of dark adaptation). A precise motorized scan (Spex monochromator) of the grating $G$ also permits one to observe the speckle variations with wavelength. Visual observation during motorized scanning at $2 \AA /$ sec was used in order to establish that the characteristic decorrelation is in the range from $30 \AA$ to $100 \AA$ for the diffusers used. The corroboration with the computed value of $80 \AA$ in Eq. (37) is excellent.

A separate series of exposures to show the averaging effect of multicolor speckle is presented in Figs. 6 through 9. Its an $8-\mu \mathrm{m}$ thick section from the optic nerve of a crayfish, prepared and stained by Roach. ${ }^{30}$ All exposures have the same magnification, and the major characteristic length of the nerve is $1 \mathrm{~mm}$. First, we should compare the appearance of this optic nerve when illuminated in laser light (no diffuser is used in this case, since it would cause speckle) and in white light as is shown in Figs. 6 and 7, respectively. It is to be emphasized that this type of comparison is essential to an understanding of the speckle problem, since the degree of difficulty in seeing things that are illuminated by a laser is highly dependent on their roughness; and of course with biological specimens, a great range of roughness is experienced. For example, from this comparison we conclude that the sample shown is not unusually diffuse, hence its visibility with laser illumination, while low, is by no means representative of a badly speckled case.

Now, a single exposure of 180-min duration for one wavelength of our 5 - $\AA$ band-limited source is shown in Fig. 8. A multiple exposure with six separate wavelengths each spaced by $300 \AA$ is shown in Fig. 9 . Great care is taken to minimize the relative motion as the grating is scanned to each new wavelength. An exposure duration of $50 \mathrm{~min}$ is used at each wavelength, the longer total exposure time being a characteristic aspect of the averaging process. A comparison of Figs. 6 and 8 shows the slight smoothing of the laser speckle caused by the finite $(5-\AA)$ width of the band-limited carbon arc source. Comparing Figs. 8 and 9 dramatically shows the improved resolution that results from using six tones of the multicolor illumination. In the holographic application, Fig. 6 shows the representative speckle for one-wavelength recording, and Fig. 9 is approximately the improvement that one would expect using six tones spanning $1500 \AA$.

\section{Conclusions}

A theoretical study of the wavelength dependence of speckle that has been so troublesome in holo-

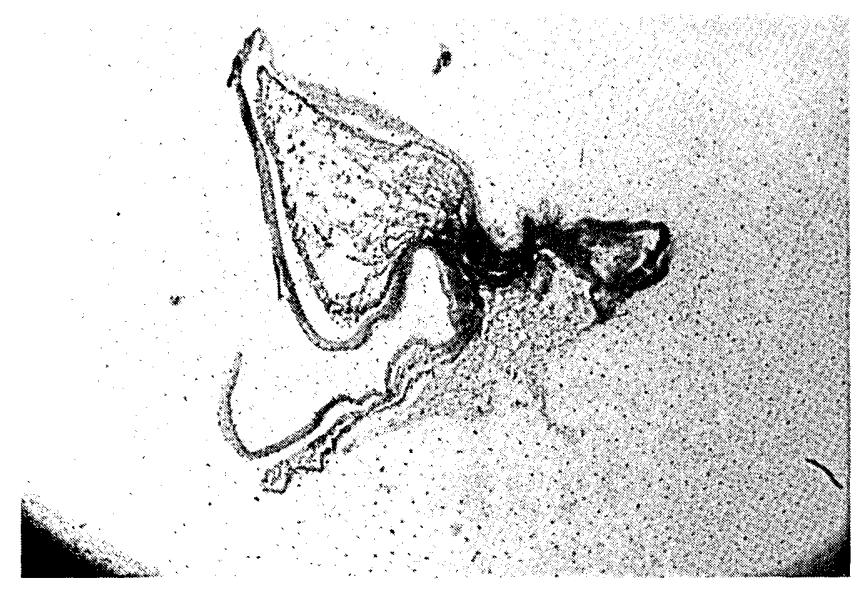

Fig. 7. Optic nerve illuminated in white light. Resolution here is much better than in the speckled image of Fig. 6. The maximum length of this specimen is approximately $1 \mathrm{~mm}$ (actual length). 


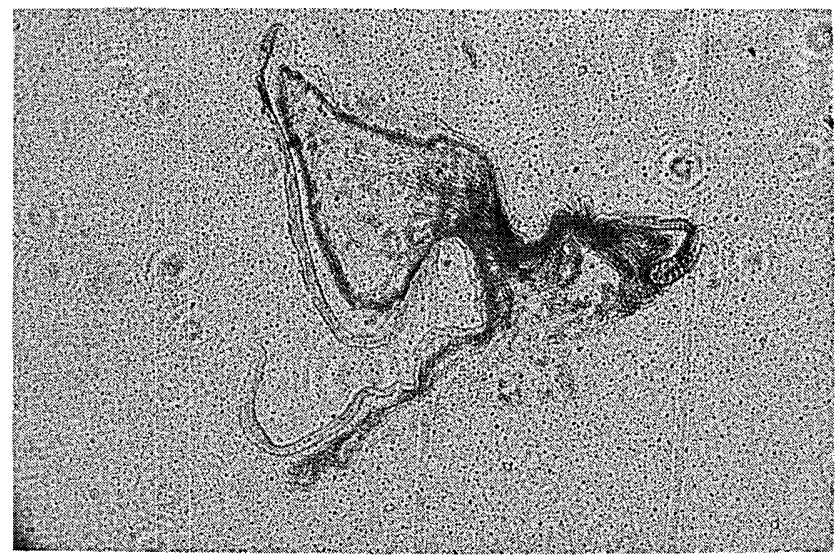

Fig. 8. Optic nerve illuminated by a collimated source at $5500 \AA$ with 5 - $\AA$ line width (180-min exposure using a high pressure mercury arc and Tri-X film). Note that the image is speckled to a slightly lesser degree than with laser illumination.
Experiments are described that verify that decorrelation results as the wavelength is scanned. In approximate terms, for highly collimated illumination, speckle is laserlike for $\Delta \lambda \leq 8 \AA$ and is greatly smoothed for $\Delta \lambda \geq 80 \AA$. Perhaps the most significant implication of this study is that it demonstrates the feasibility of eliminating speckle in holographic microscopy while still requiring only a single rapid exposure from some multimonochromatic-toned source, e.g., a dye laser.

We acknowledge that J. H. Wayland's and R. J. Bing's interest in making holograms of microcirculatory blood vessels first stimuiated our interest in speckle-free holographic microscopy; also we are pleased to acknowledge helpful discussions with $\mathrm{D}$. MacQuigg and R. B. MacAnally as well as the enthusiastic participation of Francois Bertiere in the first series of experiments with the laser sources.

This work was supported by the Air Force Office of Scientific Research.

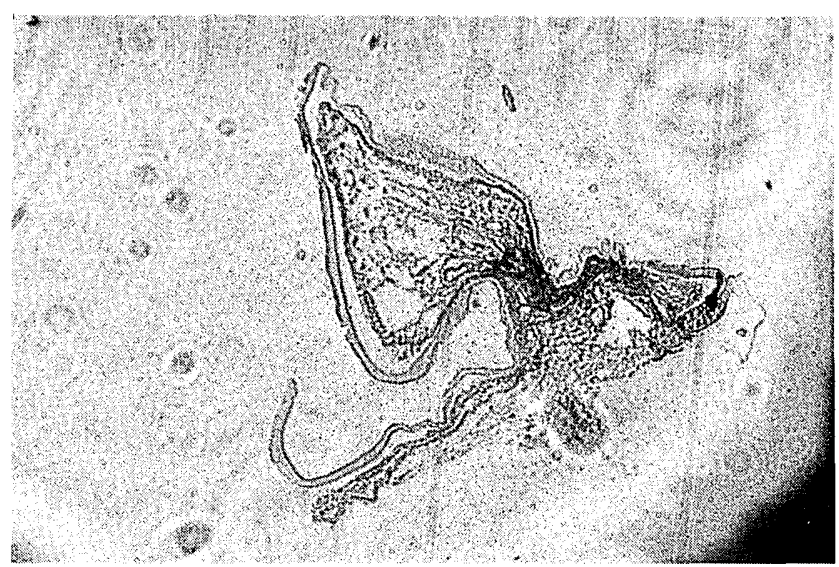

Fig. 9. Optic nerve illuminated by six separate band-limited wavelengths, spanning the spectrum from $4300 \AA$ to $5800 \AA$. Note that the resolution is considerably improved over that for a single tone as shown in Fig. 8 . The beam collimation angle of $2 \times 10^{-3} \mathrm{rad}$ is maintained throughout the series.

graphic microscopy is used to establish general criteria for the wavelength interval required in order to decouple speckle in an imaging system. A simple statistical argument is presented in analysis of Eq. (12) for the imaging of a diffuser with variable roughness; expressions for $\Delta \lambda$ to decouple with speckle or to keep it laserlike are given by Eq. (25) and (26), respectively. These are specialized for a Gaussian distribution of heights in the diffuser in Eq. (28) and then applied and discussed in an experimental context in Eq. (36).

\section{References}

1. M. v. Laue, Sitzber. Preuss. Akad. 1144, (1914), trans. by H. K. V. Lotsch.

2. J. W. Goodman, Stanford University Electronics Labs. Tech. Rept. SEL-63-140 (TR 2303-1) (Dec. 1963).

3. L. I. Goldfischer, J. Opt. Soc. Am. 55, 247 (1965).

4. L. H. Enloe, Bell Syst. Tech. J. 46, 1479 (1967).

5. W. Martienssen and S. Spiller, Phys. Lett. 24A, 126 (1967).

6. E. N. Leith and J. Upatnieks, Appl. Opt. 7, 2085 (1968).

7. H. J. Gerritsen, W. J. Hannan, and E. G. Ramberg, Appl. Opt. 7, 2301 (1968).

8. R. F. van Ligten, Opt. Technol. 1, 71 (1969).

9. R. F. van Ligten, J. Opt. Soc. Am. 59, 1545 (1969).

10. M. E. Cox, R. G. Buckles, and D. Whitlow, J. Opt. Soc. Am. 59, 1545 (1969).

11. E. Archbold, J. M. Burch, A. E. Ennos, and P. A. Taylor, Nature 222, 263 (1969).

12. M. Young, B. Faulkner, and J. Cole, J. Opt. Soc. Am. 60, 137 (1970).

13. J. C. Dainty, Opt. Acta 17, 761 (1970).

14. D. Gabor, IBM J. Res. Develop: 14, 509 (1970).

15. S. Lowenthal and D. Joyeux, J. Opt. Soc. Am. 61, 847 (1971).

16. H. H. Hopkins and H. Tiziani, Applications of Holography (Besancon Conference 6-11 July 1970), viii.

17. D. H. Close, J. Quantum Electron. QE-7, 312 (1971).

18. J. M. Burch, SPIE Devel. Hologr. 25, 149 (1971).

19. M. Elbaum, M. Greenebaum, and M. King, Opt. Commun. 5, 171 (1972).

20. N. George and A. Jain, Opt. Commun. 6, 253 (1972).

21. N. George and A. Jain, Calif. Inst. of Technol. Sci. Rept. 14, AFOSR-TR-72-1308 (1972). 
22. J. Upatnieks and R. W. Lewis, J. Opt. Soc. Am. 62, 1351A (1972).

23. Hologram volume effects and color holography are fully discussed in the following textbook and will not be treated further herein: R. J. Collier, C. B. Burckhardt, and L. H. Lin, Optical Holography (Academic Press, New York, (1971), Chaps. 9 and 17.

24. J. W. Goodman, Introduction to Fourier Optics (McGrawHill, New York, 1968), Chap. 5.

25. N. George and J. T. McCrickerd, Photogr. Sci. Eng. 13, 342 (1969).

26. As we have employed it here, the Gaussian transmission function in Eq. (2) is chosen for theoretical convenience, although for electronic optics it is physically appropriate as well. In the results it will not make any qualitative difference; in fact, in deriving the form in Eq. (15) we further approximate the actual case using a simple rect function as the impulse response of the lens.
27. In this simplification, the assumption is that the phase terms in the integrand of Eq. (3) involving the variables $x^{\prime}, y^{\prime}$ do not vary appreciably within the interval $w_{r}$. For a consideration of the extent of this type of phase variation, the reader is referred to D. A. Tichenor and J. W. Goodman, J. Opt. Soc. Am. 62, 293 (1972).

28. A. Papoulis, Probability, Random Variables, and Stochastic Processes (McGraw-Hill, New York, 1965), Chap. 8.

29. M. Born and E. Wolf, Principles of Optics (Pergamon Press, Oxford, 1970), Chap. 10.

30. The $8-\mu \mathrm{m}$ section of the optic nerve is prepared by perfusing the eyestalk in $1 \% \mathrm{~K}_{4} \mathrm{Fe}(\mathrm{CN})_{6}$ in crayfish saline; it is removed from the crayfish, soaked in saline saturated with picric acid, dehydrated in alcohol, embedded in paraffin, sectioned with a rotary microtome, stained in a Ponceau acid fushin solution; and mounted with Permount on glass.

\section{SYAPOSIU: UN MAN-PACHINE CONGUNICATION FOR SCIENTIFIC DATA HANVLIIY:}

The Task Group on Computer Use of the ICSU Committee on Data for Science and Technology (CODATA) will hold a symnosium at the University of Freiburg, Freiburg-im-Breisgau, Germany, from July 22nd to 27 th, 1973.

The purpose of the symposium is to bring together specialists concerned witi the application of computer technology to the compilation, storage, and utilization of quantitative scientific data in the physical, biological, geoloyical, and engineering sciences.

The symposium will be patterned on the Gordon Research Conferences, centering around a series of invited lectures witi opportunities provicled for small groups to deal collectively with areas of specializec interests in working seminars.

Attendence will be by invitation; interested organizations and inciviciuals are requested to write without delay to the Secretary of tire Task Group, c/o Mr. A. i. Ward, International Conferences Office, iational Research Council of Canada, Ottawa KlA ORó, Vanacia, including a brief statement of their areas of special interest. 